\title{
Desencuentro generacional o desfase metodológico en la Educación Superior, Quito, Ecuador, Marzo del 2009
}

\section{Autores:}

Dr. Efrén Horacio Vivar Reinoso

Dr. Manuel Antonio Sotomayor Martínez

Dr. Carlos Rodrigo Martínez Mosquera 


\section{Resumen}

El propósito fundamental de la investigación fue diagnosticar los principales elementos que conforman el mundo sociocultural de los jóvenes universitarios, identificando sus valores, símbolos y pautas de comportamiento en sus relaciones docente-estudiante; y, los nudos críticos que limitan el encuentro y el diálogo inter generacional para comprender las nuevas formas de conocer y aproximarse a la realidad del aprendizaje.

Se pretende poner en evidencia los problemas más urgentes que ameritan ser tomados en cuenta para innovar la praxis pedagógica en la universidad, partiendo de la superación de barreras que condicionan el diálogo pedagógico y la producción de conocimiento; potenciando la utilización de las nuevas tecnologías de la comunicación y la información para promover el cambio de mentalidad de los docentes universitarios.

La revisión bibliográfica permitió construir una visión contextualizada y prospectiva de la educación superior. Desde esa perspectiva, existe una coherencia, y una lógica relación positiva, entre el nuevo ejercicio profesional que se demanda de los docentes universitarios, y las nuevas competencias que necesita adquirir, para alcanzar un mejoramiento cualitativo de enseñanza superior. La técnica de entrevista fue aplicada a una muestra de estudiantes, seleccionada de manera intencional y de aleatoriedad simple, tanto por la cobertura de carreras como por la selección de entrevistados. La investigación de campo puso en evidencia algunos aspectos del comportamiento de la juventud frente a la vida, desde su propia perspectiva. Cómo concibe el joven su rol frente a la sociedad, frente al futuro del país y del mundo, en qué se afincan sus expectativas de cambio y transformación, cómo percibe la pluralidad de contextos y realidades socioculturales, etc. Los estudiantes, en ninguna instancia expresaron un desencuentro generacional, por el contrario muchos criterios son coincidentes con el deber ser de la universidad y del ejercicio docente. Se observa mucha valoración y expectativa respecto de los maestros en la universidad.

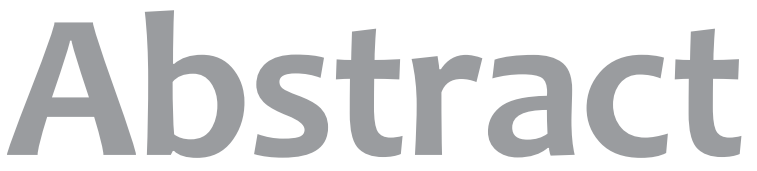

The main purpose of the research is to diagnose the principal features of the sociocultural world of university students, identifying their values, symbols and behavior guidelines in their relationship with teachers; and the critical knots that limit intergenerational match and dialog in order to understand the new ways of knowing and coming closer to the reality of learning.The investigation makes evident the most urgent problems that must be taken into account to innovate the pedagogic practice in universities, overcoming barriers that condition pedagogic dialog and the production of knowledge; enhancing the use of new communication and information technologies in order to promote the change of mindset in third level teachers.Bibliographical review allowed the construction of a contextualized and prospective vision of higher education. From this perspective, there is consistency and a logical positive relation between the new professional practice required from university teachers and the new competitions that they must achieve so as to reach a qualitative improvement in higher education.The interview technique was applied to a sample of students who were selected under an intentional and simple random procedure, considering both career coverage and interviewees selection.The fieldwork revealed some aspects of youth behavior towards life based on their point of view. That is, how young people conceive their role in the society, the country and the world, which is the basis to settle their change and transformation expectations, how they perceive the plurality of contexts and sociocultural realities, etc.Students did not talk about a 
generational mismatch. On the contrary, many criteria coincide with the duty of "being" of the university and the teaching practice. They give an important valuation and have a great expectation with regard to teachers of the three surveyed universities (UTE, PUCE, UPS).

\section{Introducción}

Desencuentro generacional o desfase metodológico en la educación superior en el Ecuador es la expresión de la preocupación de los autores, quienes constatan que en las últimas tres décadas la educación media se ha convertido en un puente obligatorio hacia la universidad. Una proliferación de universidades, el acelerado crecimiento de la población estudiantil en el nivel terciario, la amplia gama de carreras han ocasionado que muchos estudiantes deambulen los primeros años, sin lograr clarificar sus expectativas, sus opciones encontrándose además con un contexto educativo que no forma ni prepara para enfrentar los retos del futuro. Siendo tantas las preocupaciones, la central se refiere a la ruptura del diálogo entre estudiantes y docentes que se evidencia en el desfase metodológico.

Esta preocupación ya fue expresada por Ernesto Shifelbein (1993) cuando afirmó que el currículo tradicional consiste en transmitir contenidos del siglo XIX con docentes del siglo XX a jóvenes del siglo XXI. Si acaso consideramos que todavía es esencial la presencia de los educadores en la universidad hemos de reconocer la necesidad de provocar un encuentro generacional desde el conocimiento de las expectativas y demandas de los/as estudiantes universitarios, la influencia de la cultura postmoderna y la sociedad de mercado en la mentalidad juvenil, ¿Cómo perciben los jóvenes que inician la educación superior, a los docentes? ¿Cuál debe ser el perfil del docente universitario de hoy? ¿Cuál es el sentido de cambiar la metodología "de la enseñanza por una metodología del aprendizaje” en la educación superior? Y si la innovación metodológica implica cambios actitudinales y actualización docente, nos preguntamos acerca del modo de ayudar al docente a superar el miedo al cambio y a renovar su mentalidad.

El objetivo central de la investigación fue conocer la realidad socioculturaldelosylasjóvenes, queingresanalosestudiossuperiores, identificando las expectativas respecto de su formación profesional y de sus demandas respecto a la docencia y de la investigación. Para esto, los investigadores se propusieron: diagnosticar los principales elementos que conforman el mundo sociocultural de los jóvenes que ingresan a la universidad, identificando sus valores, símbolos y pautas de comportamiento en sus relaciones docente-estudiante; identificar los nudos críticos que limitan el encuentro y el diálogo inter-generacional en el contexto universitario para comprender nuevas formas de conocer y aproximarse a la realidad; $y$, diseñar una propuesta metodológica que garanticen calidad y significatividad del proceso de aprendizaje a nivel superior.

La investigación se desarrolló en tres capítulos, más las conclusiones y las recomendaciones. El primer capítulo contiene aspectos generales que constituyen los referentes del proceso investigativo; el segundo capítulo presenta varios referentes teóricos que explican los cambios socioculturales y la universidad; desde su lectura se puede comprender cómo han cambiado las sociedades, el ejercicio del poder, el avance de la tecnociencia, los cambios socioculturales configurando nuevas mentalidades. $\mathrm{Y}$ en estos contextos cambiantes se explicita el sentido de la existencia de la institución universitaria, el nuevo perfil del docente universitario y la necesidad de cambiar la mentalidad, reconstruir relaciones de diálogo entre docentes y estudiantes e innovar la metodología para salir al encuentro de las auténticas necesidades de los y las jóvenes universitarios.

En el tercer capítulo se dan a conocer algunos aspectos metodológicos, y las cifras que caracterizan la situación de las universidades en el Ecuador. Con la intención de verificar la hipótesis

\section{Tsafiqu}


central se presenta la interpretación de las percepciones estudiantiles respecto a siete aspectos de la vida estudiantil:

a) El sentido de la universidad ecuatoriana,

b) Las motivaciones de la elección de la universidad,

c) El sentido de la elección de la carrera,

d) La relación entre la universidad y el buen vivir,

e) Los docentes universitarios,

f) La pertinencia del pensum de la carrera y

g) Las innovaciones que se deberían realizar en la educación superior.

Finalmente, las conclusiones y recomendaciones permiten visualizar el sentido y el rol formador, profesional e investigativo de la educación terciaria en función del desarrollo integral del país; sobre la necesidad de comprender el fenómeno juvenil, actualizar el currículo universitario, crear estructuras que aseguren la formación y actualización docente permanente orientados hacia un cambio de mentalidad, hacia la innovación metodológica para garantizar una educación superior cada vez más significativa.

\section{Materiales, Métodos y Procedimientos}

Nos referimos acá a los actores participantes, a los métodos y técnicas de investigación, el procedimiento y los materiales.

\section{- Población de observación o muestra}

En relación a los sujetos que aportaron con información, debemos señalar que fueron estudiantes de 38 carreras diferentes, seleccionadas al azar. En el caso de UTE se han identificado las siguientes carreras: Ciencias médicas, Arquitectura, Relaciones públicas, Marketing y Publicidad, Ingeniería Mecatrónica, Gastronomía, Hotelería, Ingeniería de alimentos, Comercio exterior, Ingeniería de empresas, Turismo, Restauración y Mercadotecnia (14)

En el caso de la UPS, aparecen las siguientes carreras: Biotecnología, Filosofía y Pedagogía, Pedagogía Parvularia, Administración de empresas, Contabilidad y auditoría, Psicología, Filosofía y Comunicación social (7).

En las entrevistas cubiertas en la PUCE aparecen las siguientes carreras: Ciencias políticas, Geografía, Medicina, Contabilidad y auditoría, Negocios y relaciones internacionales (formación multilingüe), Administración, Ecoturismo, Ciencias exactas, Artes visuales (Arquitectura), Comunicación, Periodismos y comunicación, Jurisprudencia, Antropología, Sociología y Filosofía (16). Como se puede observar, existe una gama diversa de carreras en las tres universidades.

La población de estudio estuvo conformada por un total de 86 estudiantes: de la Universidad Tecnológica Equinoccial 30, de la Politécnica Salesiana 30 y de la Católica del Ecuador 26. Resultado de un proceso de muestreo intencional y a su vez, aleatorio simple, tanto por la cobertura de carreras como por la selección de entrevistados.

Para la aplicación de la entrevista se procedió a seleccionar al azar, estudiantes de los últimos años de las carreras, priorizando la condición de ser representantes estudiantiles, características de liderazgo, dirigentes estudiantiles o miembros de las directivas de los cursos.

En el levantamiento de la información mediante entrevistas grabadas, se contó con la colaboración de señoritas estudiantes, quienes fueron entrenadas y capacitadas para realizar esta tarea. Se les dio instrucciones precisas sobre:

Como iniciar la conversación de manera amigable, de tal 
forma que el entrevistado logre confianza y no tenga predisposiciones frente a un formalismo del trabajo investigativo.

- Aplicar la guía de entrevista de manera natural (lo menos formal posible). La espontaneidad y la sencillez permiten mejores resultados para la investigación que se pretende.

- Tratar al entrevistado de manera cordial y al finalizar la entrevista, agradecer su colaboración.

- Invitar al entrevistado a que participe con sus opiniones y sugerencias a través de Internet, en donde sus ideas y sugerencias serán publicadas en la plataforma virtual organizada para el efecto.

- Entregar la dirección electrónica pertinente, y si fuere necesario ayudarle para que ingrese en el espacio de “comentarios".

- Indicar que las participaciones no son anónimas, y que el sistema le pedirá su identificación. Esto, en virtud de la seriedad e importancia que se da a los diferentes aportes, para el mejoramiento de la docencia universitaria.

- Tomar en cuenta que en la entrevista sean abordados todos los temas previstos.

Gracias al levantamiento responsable, durante el proceso de entrevistas, se obtuvieron los resultados, bastante objetivos y sugerentes, que posteriormente son presentados, analizados e interpretados.

\section{- Procedimientos y análisis}

Para su procesamiento y análisis, así como la interpretación de los datos primarios, se procedió a transcribir las grabaciones de manera literal. Luego se seleccionaron las ideas principales que constan en el anexo 2, y finalmente se priorizaron ideas centrales, cuya síntesis se incluye al interior del capítulo pertinente.

De acuerdo al instrumento que consta en el anexo 1, se abordaron básicamente cinco temas a más de las referencias personales relacionadas con la universidad: 1 . dónde cursan sus estudios, 2. La carrera, 3. El semestre o años de estudio, 4. Su origen familiar y 5 . Colegio donde estudiaron el bachillerato.

Los temas abordados en las entrevistas se relacionaron con el sentido de la universidad, cómo fue su opción por la universidad, opiniones acerca de los docentes de la universidad y en especial de sus años anteriores de estudio, sobre qué innovaciones propondría para su universidad y cómo siente el ambiente universitario.

El método principal que se aplicó durante el proceso de investigación fue el hermenéutico. En la primera fase se revisó una amplia bibliografía con la intención de comprender la actualidad, pertinencia y calidad de la respuesta de la educación terciaria, a una cambiante realidad juvenil. Los contenidos de la bibliografía consultada permitieron construir una visión contextualizada y prospectiva de la educación superior, cuyo resultado está plasmado fundamentalmente en los referentes teóricos desarrollados en el capítulo anterior

Por otra parte, se analizaron las prácticas pedagógicas y antipedagógicas de los docentes universitarios, tomando como referencia los instrumentos de evaluación del desempeño docente en las universidades y justificando el clamor de los jóvenes respecto a la necesidad de cambiar de actitud y métodos por parte de los docentes universitarios. Así mismo, se realizaron entrevistas a estudiantes reconocidos por su liderazgo; la información obtenida permitió comprender algunos aspectos de la vida estudiantil.

Para profundizar la comprensión de las respuestas dadas por los estudiantes, fue necesario volver a fuentes bibliográficas actualizadas en relación a la educación superior. 


\section{Resultados y Discusión}

Los resultados se presentan desde las perspectivas teóricas y de la investigación de campo. La primera referida a la necesidad de innovar la educación superior como exigencia de respuestas significativas a los nuevos desafíos del siglo XXI; la segunda para permitir aproximarnos a la situación de la educación superior en el Ecuador y a la percepción de los estudiantes respecto a la universidad.

\section{- Desde la perspectiva teórica}

Sociedad, política, cultura y educación constituyen dimensiones dinámicas interdependientes y complementarias en la vida de los pueblos, naciones y estados porque el ser humano es constructor y a la vez, producto de la cultura, desde su capacidad de intención ha construido, en el transcurso de la historia, sistemas de relación e interdependencia, ha diseñado herramientas cada vez más complejas; ha institucionalizado aquellas experiencias exitosas para que perduren en el tiempo y ha reinterpretado en forma sistemática: creencias, saberes y valores.

Los modos de resolver problemas, de gobernar y ser gobernados, de producir bienes y relacionarse fueron cambiando desde distintas racionalidades. Estos cambios a su vez generaron nuevos modos de pensar y de actuar. Por tanto, la vida humana desde distintos órdenes ha estado marcada por los cambios cada vez más radicales y en duraciones cada vez más cortas, esta realidad es la que permite hablar de revoluciones: políticas, sociales, científicas y culturales.

\section{- Sociedad, política y cultura en cambio}

Anthony Guiddens (2001: 63) refiriéndose a la historia de las sociedades, afirma que las primitivas aparecieron hace doscientos siglos; que el desarrollo de las sociedades pre-modernas han ocupado la mayor parte de nuestro pasado. Asegura, que la agricultura solo tiene unos doce mil años de antigüedad y que las primeras civilizaciones no tienen más de seis mil años. Al organizar estos cambios en un día cósmico, asegura que "la agricultura habría aparecido a las 23:56 horas, las civilizaciones a las 23:57 horas y iel desarrollo de las sociedades modernas sólo se pondría en marcha a las 23:59 horas y 30 segundos! Según esta explicación, en esos últimos treinta segundos de la jornada humana se habrían registrado más cambios que en todas las horas previas" confirmando lo que ya había dicho Eric Hobsbawn (Boff, 2004: 29) en su obra: La era de los extremos, "cuando afirmaba que ha habido más cambios en la humanidad en los últimos cincuenta años que desde la edad de piedra"

La historia de las sociedades se divide en dos, las sociedades premodernas que datan una larga duración y las sociedades modernas cuya duración es de un poco más de dos siglos. El cambio radical estuvo marcado por la revolución industrial, la misma que tuvo como antecedente las revoluciones científicas que a su vez provocaron revoluciones políticas y culturales.

Durante las sociedades y civilizaciones agrarias, la mayoría de la población debía dedicarse al trabajo agrícola. El escaso nivel de desarrollo tecnológico liberaba de estas tareas a una mínima porción de la población. En la actualidad, el porcentaje de mano agrícola en las sociedades no industrializadas oscila entre el $64 \%$ al $91 \%$, mientras en las sociedades industrializadas, oscila entre el $2 \%$ al $6 \%$. (Guiddens, 2001: 68)

La sociedad industrial nació a partir de la revolución del mismo nombre (1770), ella produjo un salto cuantitativo en la producción pues, "nuevas fuentes de energía y de potencia, en particular el carbón y el vapor, sustituyeron el viento y el agua en la creación de máquinas que ahorraban trabajo, las cuales, a su vez, disminuyeron en forma drástica la utilización de la fuerza humana y animal y al

\section{Tsafiqu}


mismo tiempo elevaron el nivel de productividad"11

Para elevar al máximo los beneficios que se podían obtener del rendimiento mecánico se cambiaron las formas de trabajar, pues las fábricas sustituyeron a los talleres y los lugares domésticos del trabajo. Ciertamente, las condiciones físicas y de salubridad, en muchos casos, eran espantosas. Esta revolución provocó un cambio en la economía tradicional porque posibilitó el paso de un trabajo esforzado a una economía de uso intensivo del capital que requería mano de obra especializada. A su vez, el desarrollo de las fábricas en los poblados provocó una creciente migración desde las áreas rurales hacia las urbanas cambiando el sistema de relaciones sociales, aquella "intimidad de la vida rural" 22 se rompe en el área urbana donde predomina una coexistencia impersonal.

Para incrementar la productividad fueron necesarias nuevas materias primas, nuevos patrones de consumo y medios de transporte para comercializar con rapidez. La sociedad industrial permitió el nacimiento de una clase media industrial y se amplió el tamaño de la clase trabajadora, consecuentemente, cambiaron de manera radical las relaciones sociales tradicionales. Las sociedades industrializadas constituyeron la plataforma para la conformación de los estados - nación, que eran comunidades políticas con fronteras geográficas definidas, con una legislación propia que justificaba el ejercicio de amplios poderes sobre la vida de los ciudadanos.

El desarrollo de la tecnología, en la evolución de las sociedades constituyó un factor muy importante que determinó cambios en los sistemas de producción y mercado; sistemas de gobierno, tipo de propiedad, vida institucional y por supuesto, determinó la diferencia de clases sociales. Sin embargo, no se debe olvidar que el modo de gobernar estuvo regido por modos de concebir el mundo, es decir por racionalidades que en el transcurso de la historia de las sociedades,

'Spielvogel, Jackson. Civilizaciones de occidente, tercera edición, volumen B. International Thompson Editores, México, Alemania, Inglaterra, España, Australia, Estados Unidos, Francia, Chile, Brasil, Japón. 1997. P. 706/1126). también fueron cambiando.

Las relaciones sociales cambian porque cambia el ejercicio del poder. Pues el poder está estrechamente ligado al modo de entender, explicar y justificar la política; de ahí que se puede hablar de razón de la Religión, cuando la política se subordina a la Religión; razón de Estado, cuando todas las esferas de la vida humana se someten al Estado como valor absoluto; y la razón de Mercado cuando el sistema de relaciones sociales está regido por transacciones mercantiles y de consumo.

La cultura entendida como "el sistema integrado de las normas de conducta aprendidas, características del modo de vivir peculiar de un grupo social”33 es un fenómeno humano que existe gracias a la educación. Las culturas son procesos dinámicos abiertos, de ahí que podemos referirnos a una cultura tradicional, la cultura moderna, postmoderna e incluso se escucha hablar de la cultura hipermoderna.

Estas culturas no han perdido vigencia, más bien han configurado modos de pensar, sentir y actuar, es decir, han moldeado paradigmas y mentalidades que en los sujetos coexisten, muchas veces, de manera conflictiva, rompiendo el diálogo intergeneracional. La mentalidad tradicional se rige por una cosmovisión definida, única y totalizante que exige creer y obedecer a los mayores, la mentalidad moderna se fundamenta en la razón positiva, admite la pluralidad, cuestiona y valora el saber; mientras que la mentalidad postmoderna, duda del poder único de la razón, sobre valora la subjetividad y prioriza el saber estético.

Tampoco se puede negar la influencia determinante del avance de la tecnología de la información y la comunicación omnipresente en todos los órdenes de la vida y que en definitiva exige el cambio de un paradigma simple a un paradigma complejo.

Si la revolución tecnológica ha posibilitado nuevas formas

${ }^{3}$ Campos, Luis. Antropología simplificada. Imprenta FEPP, Quito - Ecuador, 1990, p.8 
de generar conocimiento, procesando la información en un circuito de retroalimentación acumulativa que se da entre la innovación y sus usos; así también se constata que los usos de las tecnologías de telecomunicación han implicado: la automatización de tareas y la experimentación de usos mediante aprendizajes por uso, mientras la reconfiguración de las aplicaciones se aprendieron haciendo y logrando estructurar redes.

\section{- La mentalidad de los jóvenes de hoy}

Muchos jóvenes sufren los efectos carenciales de la desintegración familiar, sus vidas se desarrollan de modo desequilibrado en familias monoparentales, ensambladas, sustitutas y en algunos casos donde sus progenitores no ejercieron responsablemente su rol de padres y madres. La estructura de la escuela, en sentido amplio, no ha sido pensada para potenciar el protagonismo juvenil; de cierta forma, ellos lo perciben como una carga en la que lo que más les interesa es la aceptación social de sus pares o iguales.

Muchos docentes al referirse a la respuesta de sus estudiantes afirman que su capacidad de atención en el aula es dispersa y limitada, pero son ágiles para lo que les interesa y sueñan. Tienen hipersensibilidad hacia los medios de comunicación y aprenden con una velocidad increíble el manejo de las nuevas tecnologías (NTICS). Están más preparados que los adultos, para aceptar y asimilar los cambios y las transformaciones necesarias.

Se trata de una juventud que reclama su derecho a vivir pisando fuerte en el presente y proyectarse a vivir un futuro nuevo; buscan independencia y son intransigentes, anhelan seguridad y al mismo tiempo necesitan dirección. Se sienten dueños de su vida y la viven con intensidad, desde sus criterios de valoración, que difieren del mundo de los mayores. La libertad constituye el requerimiento básico para alcanzar cualquier objetivo, aunque descalifican el sacrificio por considerar que es algo corrosivo y represivo en sus vidas.

Cuando hablan del "amor-placer", lo consideran como primera expresión de la libertad. Aceptan "la experiencia personal” como fuente y criterio de verdad y de valoración; mientras que el sentimiento de autosuficiencia y la confianza en la eficacia de la tecnología les induce a pensar que pueden todo marcando así su bagaje conductual.

Para muchos adultos, la juventud es una generación epidérmica que prefiere los caminos de la sensibilidad a los de la racionalidad; se sienten seducidos por el vértigo, la tecnología, la eficacia, la publicidad y el marketing.

La libertad de expresión con el uso de medios tecnológicos, que se van innovando día a día, y en un acelerado ritmo de vida, ha generado una devaluación de la palabra en el encuentro cara a cara, parece que desde su individualismo más les importa estar conectados que estar realmente comunicados.

El joven piensa y alardea que a él nunca le va a ocurrir ninguna desgracia; esto hace que sean más temerarios y se expongan a situaciones de riesgo que atentan a su seguridad, crecimiento personal y desarrollo humano. Cree que nadie puede sentir con tanta intensidad lo que él percibe, lo que él vive y fácilmente construye fábulas imaginarias que le permitan superar el anonimato.

Hoy los jóvenes constituyen un sector y una franja que tienen presencia en la sociedad, su principal reto es la participación en la construcción sobre todo del presente por eso esperan tener más oportunidades y quieren ser protagonistas en el desarrollo de la sociedad pero se sienten inseguros y están confundidos a la hora de enfrentar el futuro, por tanto no confían en el futuro del país. Pero, para la mayoría de los adultos, constituyen un mundo extraño; son "otros"; son diferentes pero pocos se detienen ante ellos para reconocer su alteridad, y el insondable misterio que representan los "otros".

\section{Tsafiqu}


Eljovenestálistoparahaceralgo.Suculturaesmultidimensional y cambia rápidamente. Se identifican como "emprendedores" y fácilmente se lanzan al mundo de los negocios y de la investigación. Se obnubilan por obtener rápidamente resultados y se entusiasman por los retos.

Reclaman una preparación profesional de carácter pragmático, que se les habilite para competir en el mundo laboral, quieren ser el motor en la investigación e innovación, sin dejar de ser críticos y participativos. Sueñan ser líderes y al mismo tiempo les resulta difícil cultivar un auténtico liderazgo, debido a la ausencia de referentes y modelos de vida.

Sin embargo, no se puede negar que en nuestro país se evidencia un verdadero "despertar" de sus potencialidades y su protagonismo. La fuerza de su incursión en la vida nacional y en particular en la política, constituyen un llamado a las instituciones educativas, pues no hay duda que existe en nuestra patria, un "poder y una fuerza de los jóvenes".

A partir de todo lo dicho anteriormente podríamos aproximarnos a la comprensión del impacto de todos estos cambios en una universidad anclada en la tradición, que camina de espaldas al futuro, a las culturas juveniles. En esta situación no es difícil observar practicas pedagógicas de docentes regidos por una mentalidad tradicional, positivista que intentan enseñar de modo logo céntrico a nativos digitales que viven apasionadamente el presente, que no les interesa el pasado, que sobrevaloran la subjetividad y el saber estético.

- La educación universitaria: producción de conocimiento y conciencia de sociedad

Para Alfonso Borrero (2000), el término “universitas” tiene su origen en el latín ${ }^{44}$, que según la naturaleza de los elementos, significa unidad en la diversidad. En épocas posteriores al latín, ${ }^{4}$ Op-cit. Ver volumen I, páginas 6-12. significó un gremio social expresado de diversas maneras: corpus, collegium, communio, consortium, etc. En el sentido institucional, aparecen como instituciones dedicadas a la academia; dentro de la estructura ciudadana, es una nueva dimensión política identificada como el Studium. La universidad aparece como una gran tercera fuerza frente al Imperium (poder político) y al Sacerdotium (poder eclesiástico).

En el sentido sociológico o corporativo, Borrero (2000) cita a Thomas Bender y a Walter Rüegg quienes en términos generales definen a la Universidad, "como comunidad de profesores y aprendices beneficiarios de concretos derechos autónomos para organizarse y determinar sus cursos de estudio, los objetivos de su investigación y el discernimiento de los títulos reconocidos por la sociedad..." Volumen VIII página 67.

En el sentido científico y académico, cita a Hasting Rashdall (1973, página 460 en Volumen I página 143), quien afirma que la universidad es la poseedora de todas las ramas del conocimiento, y además alude a la pluralidad y al agregado de personas. Para conocer, más profundamente, la evolución histórica de la universidad, y la diversidad de sus ramas científicas, se puede consultar la obra citada de Alfonso Borrero, Volumen I.

En nuestros días, un estudio publicado en Londres en octubre del 2006, hace referencia al Rankings Mundial de Universidades 55 , dicho estudio pone en evidencia que hoy en día la producción de conocimiento a nivel universitario está liderada por los Estados Unidos, hay una base emergente en el Asia, y hay dos continentes rezagados que son África y América Latina.

Si consideramos que el desarrollo de un país tiene su base fundamental en el sector empresarial, en el gobierno y en las universidades; dichas instancias se convierten en un trípode, que como afirma Jorge Grünberg6 ${ }^{66}$ " "ninguna de las piezas del trípode

\footnotetext{
${ }^{5}$ Publicado en "THE TIMES HIGHER EDUCATION SUPPLEMENT" de octubre 6 del 2006 , Inglaterra.
}

${ }^{6}$ Rector de la Universidad ORT, 20 de marzo de 2007, Montevideo, Uruguay. 
puede o debe ocupar el lugar de los demás. Cuando el gobierno quiere ser empresario las cosas no funcionan bien. Cuando la universidad deja de funcionar como universidad y quiere ser empresa o gobierno, tampoco las cosas funcionan bien". El gobierno tiene que brindar un marco adecuado para mantener la seguridad, garantizar una educación básica de calidad, garantizar un medio ambiente sostenible; las universidades tienen que generar conocimiento y las empresas utilizarlo para el beneficio de todos.

Si a manera de ejemplo, revisamos el acontecimiento científico-histórico de la clonación de la oveja Dolly, el científico Lan Wilmut (1999), que llevó a cabo este descubrimiento, en ese entonces era profesor en la Universidad de Edimburgo (Escocia), y ahora trabaja en Singapur; porque Singapur tiene el polo más dinámico del mundo, en biotecnología. En otras palabras podemos decir: que la tendencia actual es que el poder del conocimiento (ciencia y tecnología, que algunos autores lo denominan tecnociencia), polariza el poder económico y político en el mundo; lo que en otra hora fue la tenencia de la tierra, la tenencia del dinero, del capital, o de la mano de obra.

Es verdad que el país no tiene recursos humanos, ni institucionales, ni de mercado, ni de capital, que se hayan acumulado en fechas bastante recientes como lo han hecho otros países, por ejemplo, Singapur, Israel, Taiwán, Corea del Sur y Nueva Zelanda. Todos éstos han logrado acumular recursos de capital humano, sistemas

institucionales de promoción y de innovación en el ámbito del conocimiento, del cual nuestro país carece casi de manera absoluta. En efecto, muy pocas universidades en el Ecuador priorizan la investigación dentro de sus políticas de gestión y de búsqueda de calidad.

Sin políticas de investigación, la universidad prácticamente está perdiendo la riqueza teórica y metodológica de los esfuerzos que cada una de las unidades académicas desarrolla de manera aislada, y muchas veces repetitiva; derrochando en cierta manera sus recursos. Arocena R. y Zutz J.77 hablan de producción de conocimientos en Modo 1 y de producción de conocimientos en Modo 2, para diferenciar aquellos que son resultado de un conocimiento fuertemente disciplinario y mono institucional, que podríamos denominarlo tradicional. El conocimiento es más bien el resultado de un proceso multi y transdiciplinario, heterogéneo, poco jerárquico, estructuralmente cambiante, y de cooperación interinstitucional.

Otra manera de mirar a la universidad es como un espacio de reflexión y crítica, independiente de los poderes estructurados de la sociedad. Se le considera a la universidad como un espacio de resonancia de los problemas y conflictos sociales, como un lugar para el debate de ideas, para la comprensión de la vida; como sede de la razón, y como un espacio de formación de los cuadros profesionales que requieren los diferentes segmentos del mercado de trabajo.

Carlos de la Isla ${ }^{88}$ en su artículo, "Universidad: conciencia crítica", afirma que todos los intentos por definir el carácter específico de la Universidad, en buena parte coinciden. "La universidad es la comunidad de estudiantes y profesores que se reúnen para pensar", diría el Cardenal Neuman. Jean Paul Sastre decía: "La universidad está hecha para los hombres capaces de dudar”, "La universidad, en términos de Robert Hutchins, es el espacio escogido para meditar los problemas intelectuales del mundo" y Karl Jaspers afirma: “La universidad es el recinto sagrado de la razón"99

Como podemos observar, el atributo invariable es "pensar", en sus múltiples modalidades: meditar, dudar, analizar, razonar...etc. De allí su acertada expresión de que la universidad, es la "conciencia crítica de la sociedad".

\footnotetext{
Arocena, Rodrigo y Zutz, Judith. La Universidad Latinoamericana del Futuro. Tendencias Escenarios - Alternativas. Págs. 58 - 67. Colección UDUAL 11. Primera edición: 2001. México.

8 De la Isla, Carlos. Revista “ESTUDIOS”, No. 25, Volumen VIII, 1991, páginas 69-76. ITAM, México.

9 Jaspers Karl citado por Carlos de la Isla. Revista “ESTUDIOS”, No. 25, Volumen VIII, ITAM, México. 1991, página 69
} 
Este característico rol social que se le asigna a la universidad, constituye un aspecto fundamental que debe ser suficientemente ponderado y tomado muy en cuenta, sobre todo cuando lo vemos como una institución orientadora de la sociedad, educadora del presente para el futuro, pues acertadamente algún autor decía, “la sociedad va, hacia donde va la universidad".

Por ejemplo, los cambios en las estructuras de poder que se dan en la sociedad, son reflejos de los cambios que se dan al interior de la universidad; pues, ella es formadora de los recursos humanos que asumen el liderazgo en los diferentes ámbitos del convivir social, cultural, económico y político del Estado. Si miramos el ámbito de la competitividad, son las universidades los principales agentes, que estimulan el desarrollo social de los ciudadanos...etc. Por eso es que "la universidad debe conservar siempre su independencia, autonomía y libertad para juzgar, denunciar, anunciar e inventar, para preservar la independencia y la libertad de la sociedad”1010.

\section{- Nuevos enfoques en la pedagogía universitaria}

Luciano Bellinni, en su artículo "Educar en la Universidad de Hoy”1111, sostiene que la educación superior, poco difiere del sistema de educación media. "El profesor es dueño de su cátedra. Dicta clases, envía tareas, toma exámenes, pasa notas y allí termina su trabajo”. Incluso tiene las siguientes expresiones, mucho más fuertes para caracterizar esta realidad, y afirma:

“El sistema educativo es perverso, por tres razones: La primera, es el mismo sistema el que está centrado en las estructuras educativas, antes que en las personas. La segunda razón es que se emplea una metodología que no educa, sino que domestica. Y la tercera razón es que se utilizan contenidos que están al servicio de las mismas estructuras sociales"

\section{${ }^{10}$ Ídem.}

"Bellinni, Fedozzi, Luciano, SDB. Educar en la Universidad e Hoy, página 14.
Como quien dice, nos movemos dentro de un mismo círculo vicioso; y la única manera de salir de esta situación, nos sugiere Bellinni, es tomar a la PERSONA como centro principal del ejercicio docente universitario; antes que las ciencias, las metodologías y los contenidos.

Rafael Flórez Ochoa, en su artículo "Criterios de validación de la Pedagogía", coincide en el fondo con los planteamientos de Bellinni. Él afirma:

“Es a partir de esta posibilidad (mejoramiento del ser humano, como persona) que adquiere sentido el interrogarse pedagógicamente por alternativas conceptuales y prácticas, que permitan diseñar y planear esquemas de intervención que faciliten y afiancen el máximo desarrollo potencial humano de los individuos, siempre y cuando reconozcamos que lo más valioso en ellos es lo específicamente humano, y que tal especificidad se construye y se potencia a lo largo de la vida"1212.

Lo específicamente humano, es lo que da sentido y razón a una intervención pedagógica en todos los niveles educativos. El autor, identifica cuatro dimensiones tendenciales que potencian el eje de la evolución de la especificidad humana:

1. La universalidad que permite una mayor flexibilidad adaptativa, que a su vez se manifiesta de manera eminente en la posibilidad aprendida de interactuar en forma creadora con diferentes sistemas culturales.

2. La autonomía, es una dimensión correlativa y derivada de la universalidad; dimensión que es específicamente humana caracterizada por la actividad mental, el saber, la libertad, la capacidad de autodeterminación, constructor de sus propios valores. Es autónomo quien toma sus decisiones consciente y libremente, a sabiendas de las restricciones que surgen de la

${ }^{12}$ Ídem, p.1 
misma realidad natural y social.

3. La capacidad de acumular, analizar, sintetizar y objetivar información, es también una dimensión específicamente humana, y un campo importante con el que interactúa la pedagogía.

4. La diversidad integrada. En esta perspectiva, no caben nuevas taxonomías, sino la acentuación de las potencialidades y diferencias individuales, dentro de la humanidad única y plenamente partícipe de las mismas regularidades.

Una pedagogía universitaria, que se oriente por los principios antes señalados, garantizaría la formación de las personas, el desarrollo del pensamiento y de las capacidades innatas; el ser críticos y constructivos de una realidad. "Necesitamos hombres que piensen y no máquinas para el trabajo", así afirma Bellinni.

La hipótesis de investigación plantada en el Proyecto fue la siguiente:

“Lainnovaciónmetodológica(pasodelametodología de la enseñanza a una metodología del aprendizaje) en el nivel superior, implica cambios actitudinales (en docentes y estudiantes); y una actualización respecto de nuevos procesos de aprendizaje, nuevas formas de transmitir conocimientos, nuevas maneras de relación profesoralumno, nuevos conocimientos y destrezas a desarrollar; en definitiva, hace falta concebir una nueva forma de institución educativa”’1313 (2088 página 3 Propuesta del Proyecto)

Desde el desarrollo teórico, hemos evidenciado una demostración positiva de la hipótesis en mención. En efecto, existe una coherencia, y una lógica relación positiva, entre el nuevo ejercicio profesional que se demanda de los docentes universitarios, y las nuevas competencias que necesita adquirir,

${ }_{13}$ Ídem, p. 3 para alcanzar un mejoramiento cualitativo de enseñanza superior.

\section{- Desde la perspectiva de la investigación de campo}

Para interpretar en forma adecuada las percepciones de los estudiantes respecto a varios aspectos de la vida universitaria, es pertinente conocer en cifras la situación de la universidad ecuatoriana.

\section{o Indicadores de la educación superior en el Ecuador}

En esta última década, la educación superior en el Ecuador ha crecido en forma expansiva sin asegurar la calidad. Según la información estadística del CONESUP están registradas oficialmente 71 universidades y escuelas politécnicas; 292 institutos técnicos y tecnológicos. El $69 \%$ de estas instituciones fueron creadas en las últimas tres décadas, pues hasta 1979 existían solo 22 instituciones universitarias.

Por el sostenimiento, 29 universidades son públicas, 10 son particulares cofinanciadas y 32 son particulares autofinanciadas. De los institutos técnicos y tecnológicos 142 son públicos, 13 particulares cofinanciados y 137 particulares autofinanciados.

Las universidades y escuelas politécnicas desarrollan programas en modalidades: presencial, semi presencial y a distancia en ochenta y ocho ciudades del país. Bajo esta última modalidad, pocas universidades están incursionando en la modalidad virtual.

Actualmente se ofertan 2716 carreras, 2.242 (82,5\%) son a nivel de pregrado, de las cuales 2.030 se desarrollan en carreras de tercer nivel, 58 carreras de nivel técnico superior, 154 carreras de nivel tecnológico. Y 474 programas de postgrado que a su vez comprenden:3 doctorados(Ph.D), 279 maestrías, 98 especializaciones y 94 diplomados. La mayor parte de carreras se realizan bajo la modalidad presencial (56\%), luego está la modalidad a distancia 
(30,6\%) y la semipresencial $(13,4 \%)$

La población estudiantil universitaria, en el año 2007 ascendía a 443.509, el porcentaje de mujeres (54\%) es levemente superior al de hombres (46\%). De ellos, la mitad (55\%) estudian en universidades ubicadas en las provincias de Pichincha y Guayas.

En la última década, la población estudiantil universitaria casi se ha duplicado, pues en 1996 ascendía a 214.183 y en el 2007 fue de 443.509. Aproximadamente el $77 \%$ de ellos estudian en universidades públicas. El número de matriculados evidencia la preferencia por las siguientes áreas:

\begin{tabular}{|l|l|l|l|}
\hline \multicolumn{1}{|c|}{ AREAS } & \multicolumn{1}{|c|}{ CANTIDAD } & PORCENTAJE \\
\hline 1. & Administración y comercio & 150.172 & $33,8 \%$ \\
\hline 2. & Tecnologías & 75.886 & $17,1 \%$ \\
\hline 3. & Ciencias Sociales & 67.403 & $15,2 \%$ \\
\hline 4. & Educación & 62.975 & $14,2 \%$ \\
\hline 5. & Ciencias de la salud & 47.098 & $10,6 \%$ \\
\hline 6. & Agrícola y pecuaria & 16.197 & $3,6 \%$ \\
\hline 7. & Artes y arquitectura & 13.545 & $3,0 \%$ \\
\hline 8. & Ciencias básicas & 8.535 & $1,9 \%$ \\
\hline 9. & Humanidades y ciencias de la cultura & 1698 & $0,38 \%$ \\
\hline
\end{tabular}

El número total de docentes universitarios asciende a 22.714 de los cuales el 50.84\% tienen relación de trabajo a nombramiento y el $49.16 \%$ a contrato. Según el género, el $72,4 \%$ es masculino y $27,6 \%$ femenino. Nueve de cada diez docentes han obtenido su título de tercero y cuarto nivel en universidades del país. En el período comprendido entre el 2001 - 2007, el número de docentes se ha incrementado en un $30.91 \%$

Y respecto al número de graduados entre los años 2005 2008, ascienden a un total de 177.270 estudiantes de pregrado y 35.314 de postgrado. En las carreras de pregrado el porcentaje de mujeres (58,89\%) es mayor al de los hombres y a nivel de postgrado el porcentaje es equivalente, esto significa $50 \%$.

A nivel de pregrado, el mayor porcentaje de graduados se ubica en el área de administración y comercio (30\%), en segundo lugar está educación (20,3\%), en tercer lugar está el área de Ciencias Sociales (18,7\%), luego están las tecnologías (12,8\%) y con porcentajes inferiores a 10\% están las áreas de: Ciencias de la salud (8,8\%), agrícola - pecuaria (4,3\%), artes y arquitectura (2,6\%), finalmente está el área de humanidades y ciencias de la cultura $(0,49 \%)$.

El porcentaje de graduados a nivel de postgrado también es mayoritario en el área de administración y comercio (41,47\%), en segundo lugar está educación (25\%), luego están las ciencias sociales $(19,4 \%)$ y con porcentajes inferiores al 10\% están: las ciencias de la salud $(9,5 \%)$, tecnologías $(2,2 \%)$ y con porcentajes inferiores al $1 \%$ están: ciencias básicas (0,82\%), agrícola y pecuaria (0,76\%), artes y arquitectura (0,33\%), humanidades y ciencias de la cultura $(0,19 \%)^{1414}$.

La oferta de las carreras y postgrados responden más a estudios de mercado que toman en cuenta la gran demanda de los bachilleres pero no ha considerado las necesidades de profesionales que requiere el sector productivo del país.

El sistema educativo no tiene como referente la educación para el trabajo; en ningún nivel se atiende satisfactoriamente las necesidades básicas de aprendizaje (NBA), se maltrata social y económicamente al magisterio y no se apoya eficazmente a los institutos tecnológicos - 1515 .

Aquella escasa vinculación entre las universidades con el sector productivo, la empresa prefiere importar tecnología antes que invertir en la aplicación del conocimiento para mejorar la productividad.

La educación media se ha convertido en un puente hacia la universidad, por el que transitan más del $94 \%$ de bachilleres. La mayoría accede con graves deficiencias académicas, son producto de un sistema instruccional bancario y con escaso grado de dominio de las técnicas de estudio e investigación.

${ }^{14}$ Fuente.- CONESUP.- La educación superior del Ecuador, en cifras. Suplemento instituciona publicado en Quito - Ecuador, el 31 de octubre del 2008.

${ }^{15}$ Fuchslocher Mantilla, Guillermo. Universidades, de lo que hagan ahora depende nuestro futuro. Revista DINERS, año XXVIII, Na 294, Noviembre del 2006, p. 16-17 


\section{- El sentido de la universidad, desde la percepción estudiantil}

La pregunta ¿Cuál debería ser el aporte de la universidad a la sociedad ecuatoriana? Induce a pensar en el sentido de la universidad en la sociedad.

Desde la percepción de los/as estudiantes acerca del aporte que debe ofrecer la universidad a la sociedad se constatan visiones coincidentes que expresan la expectativa generalizada por una educación superior actualizada, equitativa y de calidad que forma personas éticas, críticas, responsables que lideren desde la investigación el desarrollo social; que sean emprendedores y que generen trabajo.

La universidad ecuatoriana debería formar cuadros de investigadores para generar conocimiento científico con sentido social. Debería invertir en la formación y actualización de sus profesores para que ellos eduquen bien a los estudiantes. Además debe estar en la vanguardia tecnológica.

La opinión estudiantil no está al margen de un deber ser de la universidad, existe concordancia conceptual y se observa una apertura y visión global tanto en el conocimiento como en el contexto internacional.

\section{- La opción por la universidad}

A la hora de elegir la universidad los jóvenes analizan varios factores: el prestigio de la institución universitaria, el ambiente, las carreras que oferta y los costos de la colegiatura. $\mathrm{Y}$ al preguntarles ¿Cuál fue el principal motivo por el cual escogió la universidad dónde realiza sus estudios?, la mayoría afirma haber tenido en cuenta la calidad de la atención educativa que la institución ha ofrecido durante años y que sin embargo continua vigente mereciendo el reconocimiento internacional y nacional. Prestigio social respaldado en las referencias dadas por personas significativas.
Otro factor de la preferencia es saber que en aquella universidad existe la carrera que busca el estudiante y que tanto el pensum es pertinente y el cuadro de docentes asegura experiencias de aprendizaje significativos. Para muchos, es muy importante la accesibilidad económica y más aún cuando los estudiantes solventan su colegiatura.

Para algunos también es importante sentir afinidad con la filosofía institucional porque ésta se expresa en políticas que contribuyen a la construcción de un ambiente y estilo propio

\section{- Sobre la elección de la carrera}

Se pensaba que era creciente el número de estudiantes que ingresa a la universidad sin haber decidido con suficiente claridad su carrera, y que debido a la dependencia económica se somete a la decisión de sus progenitores quienes aseguran pensando en el futuro exitoso de sus hijos e hijas. Por esta razón se les ha preguntado ¿Quién eligió la carrera que usted está estudiando? Y algunos afirman que es el rumbo que está tomando su vida el que ha determinado la elección de la carrera que está siguiendo; otros afirman que eligieron la carrera por ser nueva, interesante y porque les gusta. Además, porque cuentan con el apoyo incondicional de su familia.

En casi la totalidad de los casos entrevistados, existe una decisión personal que demuestra una reafirmación de la personalidad de los estudiantes universitarios; conviene tener en cuenta que los entrevistados pertenecen a universidades privadas; el resultado sería diferente si los entrevistados proviniesen de universidades públicas.

\section{- La universidad y el buen vivir}

La percepción estudiantil respecto a las relaciones entre la formación universitaria y la calidad de las condiciones de vida se indagó a través de la pregunta ¿Piensas qué los estudios universitarios son indispensables para vivir bien? A la cual muchos estudiantes 
respondieron que los estudios universitarios son indispensables, pero insuficientes, porque el mercado laboral es competitivo y exige no solo elevar el nivel profesional realizando estudios de postgrado, sino el desarrollo de nuevas competencias que posibiliten la estabilidad y mejores oportunidades.

Otros ratifican cuán indispensable es la profesionalización de personas emprendedoras con sensibilidad social y algunos señalan que la universidad no es el único contexto donde se dan lugar los aprendizajes y que vivir bien no se reduce a tener más.

\section{- Acerca de los docentes y la docencia universitaria}

Para recabar la percepción de la juventud universitaria acerca de los docentes y la docencia universitaria se realizaron tres preguntas, la primera fue la siguiente: sabiendo que el desarrollo de las nuevas tecnologías de la información y la comunicación se están implementando en las universidades, ¿Cree qué llegará el día en que la presencia de los docentes no sea indispensable? Y casi todos consideran que el docente universitario tiene un rol muy importante en la formación universitaria y que la tecnología jamás reemplazará la labor del docente. Muchos hacen referencia a la experiencia y que la tecnología es un instrumento que debe ser utilizado con mayor eficacia en la formación de los profesionales en las diferentes carreras.

Actualmente la formación del talento humano en todos lo campos no pueden estar al margen del avance de la ciencia y la tecnología. Los currículos en todos los niveles educativos deben poseer alta flexibilidad, de manera que la dinamia de transformación sea adecuadamente asimilada por los miembros de nuestra sociedad. La generación del “doble clic”, como lo denominan algunos autores, o los nativos digitales, como los llama Marc Prensky ${ }^{1616}$, deben

${ }^{16}$ Marc Prensky, es un líder del pensamiento, conferencista, escritor, consultor y diseñador internacional de juegos en las áreas críticas de la educación y del aprendizaje. Él es autor de "Digital GameBased Learning" (McGraw-Colina, 2001), fundador y CEO de Games2train, una compañía de aprendizaje basado en el juego, y fundador de The Digital Multiplier, una organización dedicada a eliminar del mundo la división digital en el aprender. encontrar la respuesta oportuna, ágil y veraz, en todas las instancias del saber universitario.

Ala pregunta ¿En qué caso, un docente universitario merecería su respeto? han asegurado que los docentes se ganan el respeto cuando son íntegros y de alta calidad humana, cuando se nota que aman lo que hacen sin reducirse a individuos asalariados, porque al estar equilibrados son amables en el trato, evidencian coherencia entre lo que dicen y hacen. Este modo de ser genera un clima de respeto en el que todos aprenden de todos.

Un docente en todo el sentido de la palabra, es quien respeta a todos sin interiorizar, enseña sin arrogancia, alienta, corrige y evalúa con justicia etc. El respeto debe estar presente siempre, en todo lugar y relación con el docente. Y en cuanto a su desempeño es fácil darse cuenta de su experiencia, del dominio de los conocimientos, es decir, cuán preparado está para impartir su cátedra y llegar a sus estudiantes. Si es innovador y planifica siempre las clases con la intención de contribuir para que todos crezcamos.

El valor del respeto aglutina otros valores como lo está manifestando en las respuestas de los estudiantes. En efecto, aparecen otros valores como la experiencia, la justicia, la humildad, de trato amable y equilibrado, la coherencia entre lo que dice y lo que hace, el ejemplo, el amor a la profesión o al ejercicio profesional, la igualdad de criterios, integridad, preparación y competencia profesional, etc. Son los tantos aspectos que los estudiantes valoran en sus maestros.

En otras palabras, los estudiantes demandan respeto, eticidad y profesionalidad de parte del docente.

Cuando se les ha preguntado, si al terminar la carrera universitaria, y las autoridades le ofrecieran una cátedra, ¿Aceptaría el cargo? ¿Por qué? la mayoría de los estudiantes entrevistados coinciden que no aceptarían el cargo como docentes de la Universidad, pues admiten que un docente necesita tener tanto las 
aptitudes como habilidades para poder transmitir satisfactoriamente los conocimientos a los jóvenes; además, toman en cuenta que el trabajo del docente no es bien remunerado. Y quienes aceptarían el cargo, lo harían por gratitud a sus educadores y a la Universidad; también por el interés de evaluar el nivel de sus conocimientos e irlos actualizando.

\section{- Acerca de las innovaciones en la educación superior}

Respecto a las innovaciones a realizarse en la educación superior se ha preguntado: si usted fuese el rector de la universidad ¿Qué cambiaría para que la Universidad alcance la excelencia?

Casi todos los estudiantes universitarios afirman que existe demasiada burocracia, cambiarían todos los trámites innecesarios, harían una rendición de cuentas claras para evitar malos entendidos, derroche de dinero e injusticias en el pago a los docentes. Permitirían a los estudiantes tener voz y voto en la elección de rectores, profesores y otras decisiones que involucren el avance de la universidad. Además financiarían proyectos e investigaciones para continuar recopilando información, al mismo tiempo sienten una necesidad de vivir o sentir más de cerca de la realidad nacional actual. Las posibles reformas que sugieren tienen relación con la gestión institucional, con la calidad de las relaciones interpersonales y con la actualización de la misión de la universidad.

Pensando en el pensum de la carrera que estudia, señale una o dos materias que suprimiría. En las universidades Católica y Salesiana la mayor parte de su población estudiantil opinan que se debe suprimir toda materia relacionada con el catolicismo (Religión, Catequesis, Biblia, Pastoral y Antropología cristiana) ya que consideran que es una forma de irrespetar sus creencias individuales, ya que no todos son católicos y consideran que se pretende implantar la ideología de estas universidades. Mientras tanto los estudiantes de la UTE consideran innecesarias las materias relacionadas con las ciencias humanas y no argumentan sobre ello.
Por otra parte, se evidencia en los estudiantes una aparente ingenuidad al aparentar un desconocimiento de la filosofía institucional, pretendiendo de ella una oferta que responda a conveniencias individuales.

¿Cuándo ingresaste a la Universidad qué fue lo que más te impacto? La mayoría de estudiantes opinan que les impacto la cordialidad y apertura de los docentes lo cual les ha permitido tener una relación más cercana a ellos pues sus ideas han sido valoradas, escuchadas y respetadas por sus enseñantes esto les evidencia la gran calidad humana y profesional de sus docentes. En cuestión de horarios afirman que les costó un poco acoplarse a ellos pues ahora el estudio les ocupa mayor cantidad de su tiempo y no se les facilita el poder realizar otra actividad como trabajar. También se refieren mucho a que al ingresar a esta nueva etapa de su vida tuvieron que adquirir mayor responsabilidad y libertad ya que no existe un acompañamiento ni seguimiento de parte de un adulto hacia ellos como lo era en el colegio esto les demanda mayor discernimiento.

$\mathrm{Y}$ respecto a lo que más les molesta de sus compañeros/as, la mayoría expresa una profunda inconformidad con el ambiente académico, refiriéndonos exclusivamente a los compañeros de facultad pues no observan en ellos compromiso, responsabilidad y compañerismo. Algunos no respondieron a esta pregunta porque se consideran personas tolerantes e incluso unos pocos no se han dado cuenta de nada malo.

\section{Conclusiones y Recomendaciones}

\section{Conclusiones}

1. Desde la perspectiva del desarrollo teórico, se ha evidenciado una demostración positiva de la hipótesis antes mencionada. En efecto, existe una coherencia, y una lógica relación positiva, entre el nuevo ejercicio profesional que se demanda de los docentes universitarios, y las nuevas competencias que 
necesita adquirir, para alcanzar un mejoramiento cualitativo de enseñanza superior.

2. Desde la perspectiva de los roles sociales que la Universidad tiene en el contexto nacional, se puede deducir que tanto el país como sus universidades carecen de una visión adecuada de los roles o funciones que deben cumplir. Esto es un problema que se deriva de una cantidad de factores culturales y sociales, que se deben modificar si es que se quiere desarrollar adecuadamente una universidad para la nueva sociedad del conocimiento.

3. El hecho de afirmar que las universidades "deben producir aprendizaje", cambia la responsabilidad hacia la institución. Institución que prioritariamente está sustentada por los estudiantes y los docentes. Los estudiantes son lo coproductores en el aprendizaje, ellos pueden y deben tomar la responsabilidad de su propio aprendizaje.

4. En este juego del aprendizaje, los dos agentes (estudiantes y docentes) asumen la responsabilidad por el mismo resultado, sin que ninguno tenga un control completo de todas las variables. Es la sinergia grupal la que dará cada vez mejores resultados en la educación universitaria.

5. Desde la perspectiva de la percepción de los estudiantes, en ninguna instancia se percibe un desencuentro generacional, por el contrario muchos criterios son coincidentes con el deber ser de la universidad y del ejercicio docente. Por ningún concepto creen que el docente sea sustituido, ni su rol fundamental va a cambiar en el corto plazo. Por el contrario se observa mucha valoración y expectativa respecto de los maestros en la universidad.

6. La referencia a los valores en el proceso de formación profesional es relevante, se menciona además, la calidad de la educación como responsabilidad de la universidad como orientadora de la sociedad y como líder en el conocimiento, en el desarrollo tecnológico y en la investigación. Todos estos aspectos coinciden con los planteamientos teóricos desarrollados en el capítulo anterior.

7. Elementos comoel prestigio, la calidad, la excelencia, el carisma institucional, etc. son ideas que surgen frecuentemente en las entrevistas a los estudiantes de las tres universidades.

8. La movilidad social y económica que ofrece el sistema educativo sobre todo en el tercer nivel, es claramente percibida por los estudiantes universitarios. El ascenso social y el mercado de trabajo, parecen ser los referentes que más les preocupa a los estudiantes.

9. Muchos estudiantes hacen referencia al docente como un referente o ejemplo como persona, como profesional y como ciudadano. El es un guía del aprendizaje y de la formación de la juventud. Demuestran poco interés por ejercer la docencia universitaria.

\section{Recomendaciones}

1. Como las tecnologías están revolucionando todos los ámbitos del saber y del convivir humano (y la educación no se encuentra al margen de dichas influencias), una excelente estrategia para mejorar la docencia en la universidad es ir progresivamente incorporando las nuevas tecnologías de la información y de la comunicación, al quehacer del aprendizaje y la investigación universitaria.

2. Imaginarse una universidad sin paredes, donde puedan elegirse carreras, programas, cursos y profesores; donde se pueda seleccionar bibliotecas, museos, libros de textos, resultados de investigaciones de todo el mundo, documentos informativos de todo tipo, sobre todo académico; es 
prácticamente hoy, una realidad que se optimiza cada vez más, pues se tienen las condiciones para ello.

3. Los directivos y docentes de la universidad, debemos buscar para la educación, nuevas metodologías, nuevas prácticas sistémicas de Interaprendizaje, maneras distintas de convivir con las autopistas de la información y tener algo que entregar para las nuevas generaciones. La renovación de las metodologías educativas en la Universidad, constituye garantía de calidad; la participación de los estudiantes en el sistema universitario, los planes estratégicos universitarios y los sistemas de ayudas para los estudiantes, son otros aspectos que deben igualmente incorporarse a la gestión universitaria.

La idea de agregar valor al cumplimiento de la misión universitaria, con tecnologías nuevas, supone ciertas condiciones que la Conferencia de Rectores de Universidades Españolas (CRUE, 1997) ${ }^{1717}$, las recoge de la siguiente manera:

a. Acceso de estudiantes y profesores a la infraestructura tecnológica apropiada.

b. Desarrollo de metodologías docentes para el aprovechamiento de las TICs.

c. Desarrollo de materiales y recursos didácticos de apoyo a la docencia basados en TICs.

d. Formación del profesorado y de los estudiantes, abiertos a la utilización de las TICs.

e. Fomento del uso de las Tecnologías de la Información y las Comunicaciones en las actividades de formación y de aprendizaje universitario.

${ }_{17}$ Citado por Jesús Salinas, en su ponencia "Rol del profesorado universitario ante los cambios de la era digital". Representante de la Universidad Islas Belares. Caracas, Universidad Central de Venezuela. Julio, 1999 .

\section{Bibliografía consultada}

1. AAVV. Universidad: entre la modernización y el mercado, revista Universitas, año VI $-\mathrm{N}^{\mathrm{a}} 9$, Universidad Politécnica Salesiana del Ecuador, Cuenca, 2007, pags.232

2. Boff Leonardo. Ética y moral. Sal Terrae, Santander Colombia, 2004, págs. 134

3. Campos, Luis. Antropología simplificada. Imprenta FEPP, Quito - Ecuador, 1990, p. 170

4. Guiddens Anthony. Sociología, cuarta edición. Alianza editorial. 2001, págs. 944

5. Schiefelbein Ernesto. En busca de la escuela del siglo XXI. UNESCO/UNICEF, 1993, págs. 49

6. Spielvogel, Jackson. Civilizaciones de occidente, tercera edición, volumen B. International Thompson Editores, México, Alemania, Inglaterra, España, Australia, Estados Unidos, Francia, Chile, Brasil, Japón. 1997. p. 1126).

\section{El Investigador}

Dr. Efrén Vivar Reinoso evivar@ute.edu.ec

Licenciado en CIED, Pontificia Universidad Católica del Ecuador, 1976

Doctor en CIED, Pontificia Universidad Católica del Ecuador, 1977

Licencia en Teología Past., Pontificia Studiorum Universitas Salesiana, 1986 